\title{
Wenn Kinder Schmerzen haben
}

\author{
Stefanie Zang
}

Schmerzen bei Kindern können die unterschiedlichsten Ursachen haben. Ihnen auf den Grund zu gehen ist oft schwierig, aber immens wichtig, denn die Folgen von unbehandelten Schmerzen können die gesamte kindliche Entwicklung bedrohen. Zwei Expertenstandards dienen als Grundlage für ein professionelles Schmerzmanagement.

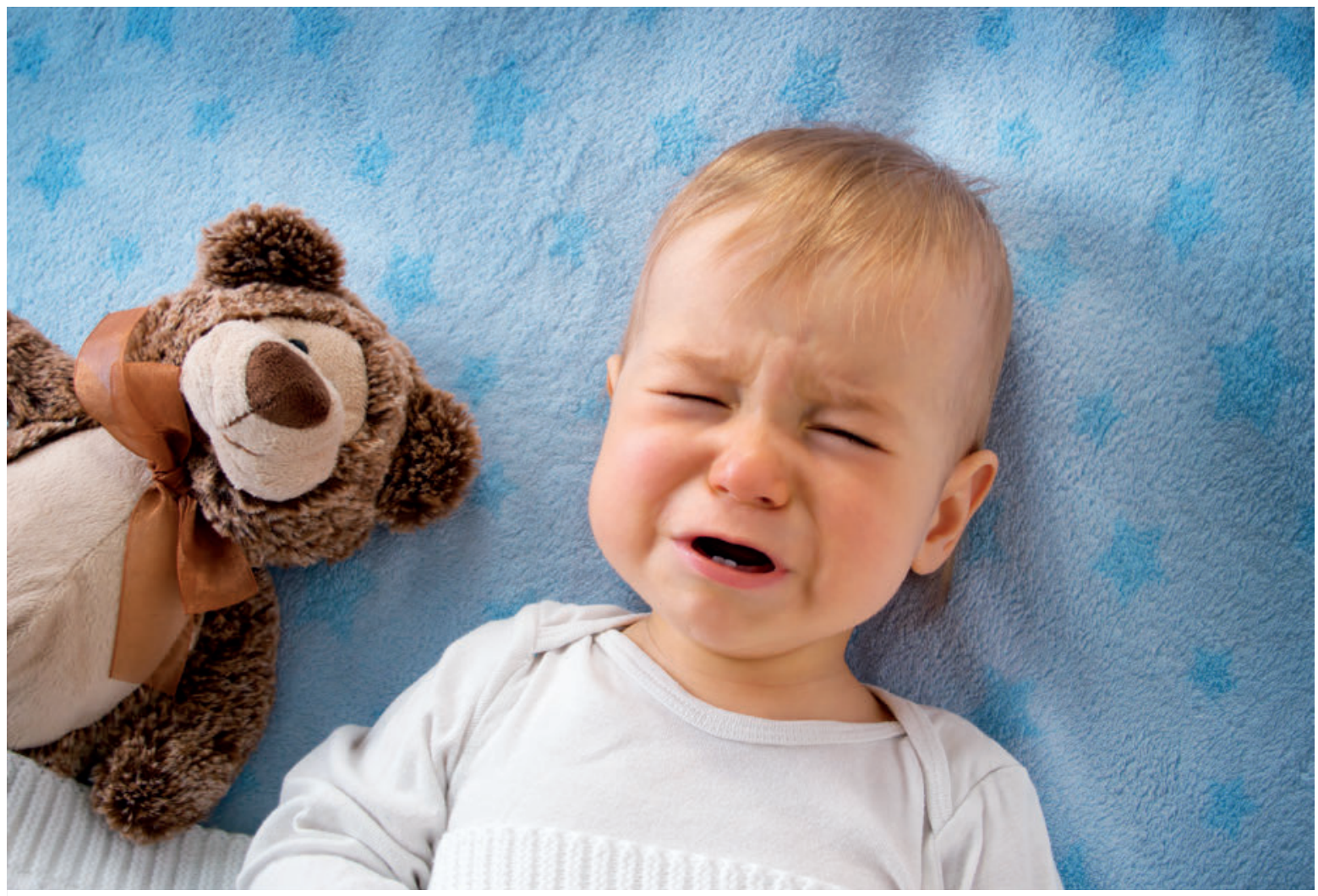

Bei der Einschätzung von Schmerzen bei Kindern gilt es, die kindspezifischen, entwicklungsbedingten Besonderheiten der Kinder zu berücksichtigen und in die pflegerische Schmerzeinschätzung einzubeziehen. (Quelle: candy1812_AdobeStock; Symbolbild)

Schmerz ist ein Phänomen, das untrennbar mit dem Leben verbunden ist - auch bereits im Kindesalter. Jedoch wurden Schmerzen bei Kindern lange Zeit vernachlässigt und häufig sogar komplett ignoriert. Bis weit in die 80er-Jahre des 20. Jahrhunderts standen Aussagen wie „Neugeborene und Säuglinge sind aufgrund ihres unreifen Nervensystems schmerzunempfindlich, Kleinkinder vergessen den Schmerz sofort wieder" oder „Das Risiko einer Schmerztherapie bei Kindern übersteigt den möglichen Nutzen " für einen professionellen Handlungsansatz in der Behandlung von kranken Kindern [1][2]. Heute wissen wir, nicht zuletzt aufgrund der bahnbrechenden Forschungsarbeiten von Anand [3], dass Kinder unabhängig ihres Alters Schmerzen empfinden können; die Wichtigkeit einer adäquaten Schmerztherapie sowie eines notwendigen pflegerischen Schmerzmanagements sind unbestritten. Insbesondere für die Klientel der häuslichen Kinderkrankenpflege - chronisch kranke, pflegebedürftige Kinder/Jugendliche mit und ohne Behinderungen - ist ein professioneller Umgang mit dem Pflegeproblem Schmerz von zentraler Bedeutung.

Ein nachfolgender Blick auf relevante Forschungsergebnisse zu Schmerzen bei Kindern und Jugendlichen soll diese Aussage untermauern. 


\section{Verschiedene Schmerzarten}

Schmerzen gehen einher mit Verletzungen oder Krankheiten; bei Kindern und Jugendlichen stehen diese meist in Verbindung mit medizinischen Interventionen wie Impfungen, Blutentnahmen, Zahnarztbesuchen oder Operationen. Infektionskrankheiten wie Otitis media oder Stomatitis aphtosa, aber auch Stürze von der Kommode oder mit dem Fahrrad begleiten das Kindesalter und gehen mit akuten Schmerzen einher [4][5].

Ein ernst zu nehmendes Problem ist - analog zu den Erwachsenen - die fortschreitende Anzahl von Kindern und Jugendlichen mit chronischen Schmerzen, also Schmerzen, die bereits länger als drei Monate bestehen und somit ihre Warn- und Schutzfunktion verloren haben [6]. So lebten im Jahr 2015 nach Aussagen des Deutschen Kinderschmerzzentrums in Deutschland ca. 350.000 Kinder und Jugendliche, die an chronischen Schmerzen leiden - mit steigender Tendenz. Hierbei handelt es sich um ein breites Spektrum an unterschiedlichen Erkrankungen, insbesondere chronische Bauch- und Kopfschmerzen, neuropathische Erkrankungen, juvenile Arthritis oder Fibromyalgie, schwere Zerebralparese sowie somatoforme Schmerzstörungen [7]. Auch die repräsentativen Ergebnisse des Kinder- und Jugendgesundheitssurveys (KIGGS) zeigen, dass 71 Prozent der knapp 15.000 erfassten Kinder und Jugendlichen im Alter von drei bis 17 Jahren in den letzten drei Monaten über Schmerzen berichteten, mit zunehmender Schmerzprävalenz bei steigendem Alter. Häufigste Schmerzlokalisationen waren hierbei Kopf, Bauch und Rücken [8][9].

Als eine besonders vulnerable Gruppe gelten Kinder und Jugendliche mit schwersten Mehrfachbehinderungen. Aufgrund ihrer neurodegenerativen, genetischen oder postinfektiösen Grunderkrankung werden bei ihnen häufig schmerzhafte chirurgische Operationen wie Kontrakturoperationen oder Zahnextraktionen durchgeführt. Komorbiditäten wie Epilepsie, Spastik, Refluxösophagitis, Obstipation, Gallen- und Nierensteine oder eine Aspirationspneumonie sind häufige Begleiterscheinungen einer Mehrfachbehinderung, die ihrerseits Schmerzen verursachen. Auch notwendige pflegerische Maßnahmen wie regelmäßige Bewegung und Transfermaßnahmen, das Anlegen von Orthesen oder die tägliche Mund- und Zahnpflege können bei diesen Kindern Schmerzkrisen auslösen [10].

\section{Folgen einer ausbleibenden Schmerzbehandlung}

Die Folgen von unbehandelten Schmerzen im Kindesalter sind vielfältig. Sie verursachen nicht nur momentanes Leid, sondern bedrohen die gesamte kindliche Entwicklung. Heute wissen wir: Je mehr schmerzhafte Erfahrungen ein Früh- und Neugeborenes erlebt hat, desto langsamer und eingeschränkter entwickeln sich seine geistigen und motorischen Fähigkeiten [1]. Gerade rezidivierende Schmerzen im Kindesalter gehen mit einer erhöhten Ängstlichkeit und Depressivität, physischen und psychosozialen Einschränkungen und vielen Schulfehltagen einher [11]. Zusätzlich wirken sich Schmerzen negativ auf Appetit, Stimmung, Schlaf- und Spielverhalten aus; ebenso wird die kindliche Neugier dadurch gehemmt [12].

Neben (teils auch lebensbedrohlichen) körperlichen Symptomen wie eingeschränkte Atem- und Nierenfunktion, eingeschränkte Verdauung, verminderte Sauerstoffsättigung des Bluts, ein erhöhter Grundumsatz inkl. Immunsuppression, Schock etc. [12] führt eine unzureichende Schmerzlinderung zu veränderten Schmerzreaktionen bzw. einer niedrigeren Schmerzschwelle, die Kinder werden also zunehmend schmerzempfindlicher, der Bedarf an Schmerzmitteln steigt [4]. Und nicht zuletzt können extreme Schmerzerfahrungen im Kindes- und Jugendalter zu einer veränderten Schmerzverarbeitung, also zur Ausbildung eines Schmerzgedächtnisses führen [4][11].

Zusammenfassend wird deutlich, dass starke akute, rezidivierende und chronische Schmerzen bei Kindern und Jugendlichen zu Beeinträchtigungen der Lebensqualität und zu erheblichen körperlichen, psychologischen und sozialen Einschränkungen führen.

\section{Professionelles Schmerzmanagement}

Vor dem Hintergrund dieser Schmerzfolgen eröffnet sich die Bedeutung einer zügigen Schmerztherapie sowie eines umfassenden Schmerzmanagements seitens der (häuslichen) Kinderkrankenpflege. Für die Umsetzung bilden die beiden Expertenstandards des Deutschen Netzwerks Qualitätssicherung in der Pflege (DNQP) zu akuten und chronischen Schmerzen [4][6] bzw. deren Adaption für die Kinderkrankenpflege durch den Bundesverband häusliche Kinderkrankenpflege e. V. (BHK) sowohl die geforderte rechtliche als auch pflegewissenschaftliche Grundlage [9][13]. Zentrale Aussagen aus diesen Standards sollen nachfolgend kurz skizziert werden.

Der Kern eines jeden professionellen Schmerzmanagements - so auch in der häuslichen Kinderkrankenpflege ist die Schmerzeinschätzung bzw. Schmerzerfassung, mit der jedes Schmerzmanagement beginnt. Hier zeigt sich bereits eine erste Besonderheit in der Pflege von kranken Kindern.

Schmerz ist ein komplexes Geschehen - spätestens seit der Veröffentlichung der Gate-Control-Theory von Melzack und Wall (1965) ist dieser Aspekt unumstritten: Schmerz umfasst nicht nur physische, sondern auch kognitive und soziale Komponenten [5]. So definiert die International Association for the Study of Pain (ISAP) Schmerz als eine „unangenehme sensorische und emotionale Erfahrung in Verbindung mit einer tatsächlichen oder möglichen Gewebe- 
schädigung" [4]. Schmerz ist jedoch vor allem eine individuelle und subjektive Empfindung, was sehr praxisnah in der Schmerzdefinition von McGaffery et al. beschrieben wird: „Schmerz ist das, was der Betroffene über die Schmerzen mitteilt, sie sind vorhanden, wenn der Patient mit Schmerzen sagt, daß er Schmerzen hat. “ [14]

Beide Definitionen thematisieren Gefühle und Erfahrung sowie die Fähigkeit, Schmerzen auszudrücken. Hier wird deutlich, warum Schmerzen u. a. bei Kindern eine besondere Herausforderung darstellen. Denn nicht alle Kinder können ihre Schmerzen adäquat verbal mitteilen - sie sind dafür zu jung, zu krank, zu ängstlich, körperlich oder kognitiv zu eingeschränkt, und es fehlt ihnen sicherlich auch an einer gewissen Lebenserfahrung, Gefühle eindeutig unterscheiden zu können [12].

\section{Erster Schritt: Schmerzerfassung}

Die Schmerzerfassung bei Kindern und Jugendlichen ist somit eng verbunden mit dem jeweiligen körperlichen, geistigen und emotionalen Entwicklungsstand. Pflegende sind beispielsweise bei Säuglingen oder Kleinkindern weniger auf verbale Zeichen als auf die Beobachtung und Wahrnehmung von schmerztypischen Verhaltensmustern angewiesen. Auch die Fähigkeiten, sich mit dem Konzept Schmerz auseinanderzusetzen, Ursachen zu erkennen, ihn konkret zu kommunizieren und darauf zu reagieren, verändern sich im Zuge des Älterwerdens [11]. Und nicht zuletzt lernen Kinder am Modell ihrer Eltern - so beeinflussen auch bei dem Thema Schmerz die Reaktionen der Eltern oder nahen Bezugspersonen das kindliche Schmerzverhalten [5].

Diese kindspezifischen, entwicklungsbedingten Besonderheiten gilt es als Pflegende in der häuslichen Kinderkrankenpflege zu reflektieren, zu berücksichtigen und in die pflegerische Schmerzeinschätzung zu integrieren - insbesondere, da nicht nur bei Erwachsenen, sondern bereits bei Kindern das Thema Selbsteinschätzung vor Fremdeinschätzung als Goldstandard der Schmerzerfassung definiert wird [11].

So beinhaltet jede Schmerzerfassung eine umfassende Schmerzanamnese, d. h. Einschätzung von

- Schmerzsituation (Schmerzlokalisation, -ursache, -beginn, -dauer, -qualität, -auslöser, akut/chronisch, stabil/instabil etc.),

- Schmerzintensität (mittels valider Messinstrumente, ausgewählt nach Alter, Schmerzursache und kognitiver Fähigkeiten, z. B. kindliche Unbehagensund Schmerzskala - KUSS für Neugeborene bis Ende des 4. Lebensjahrs; Faces Pain Skale Revised - FPS-R zur Selbsteinschätzung für Kinder zwischen dem 4. und 8. Lebensjahr; Non-Communicating Children's Pain Checklist - Revidierte Version - NCCPC-R für Kinder mit Schwerst-/Mehrfachbehinderung u.v.a.m.)
- Schmerzvorgeschichte (frühere Schmerzerfahrungen, Schmerzverhalten, Reaktionen der Eltern, Auswirkungen auf den Familienalltag etc.),

- Stabilisierenden/destabilisierenden Faktoren (Tagesrhythmus, Zuwendung, Ablenkung, Schmerzmedikation, Bewegung/Ruhe, Langeweile, Schlafentzug, Hektik, Lärm, Schulprobleme etc.). [4][6][9][13]

Die Einbeziehung der Eltern und Bezugspersonen ist hierbei unabdingbar, insbesondere bei Kindern und Jugendlichen mit schwersten Mehrfachbehinderungen. Diese verfügen nur eingeschränkt über schmerztypische Verhaltens- und Kommunikationsmuster [10], jedoch sind Schmerzen häufig der Grund für deutliche Verhaltensänderungen dieser Kinder, die die Eltern meist zu einem frühen Zeitpunkt erkennen und benennen können.

\section{Zweiter Schritt: Schmerztherapie}

Ist der Schmerz des Kindes oder Jugendlichen erfasst, muss dieser zügig behandelt werden, die zweite tragende Säule eines Schmerzmanagements ist somit die Schmerztherapie. Gemeinsam mit Kind, Eltern, Kinderärztin und ggf. einer pflegerischen Schmerzexpertin sowie weiteren Therapeuten wird ein individueller Behandlungsplan erarbeitet. Im Mittelpunkt dieses Behandlungsregimes steht die medikamentöse sowie nicht-medikamentöse Schmerztherapie. Letztere ist gerade bei Kindern und Jugendlichen von großer Bedeutung, da nicht-medikamentöse Maßnahmen kaum Nebenwirkungen haben und meist einfach anzuwenden sind. So zeigen beispielsweise folgende Maßnahmen eine schmerzlindernde Wirkung:

- Ablenkung (durch Seifenblasen, Filme, Spiele, Handpuppen, Luftblasen, Bilderbücher, Denkaufgaben etc.)

- Entspannung (durch körperliche Nähe, Zuwendung, Abbau von Angst und Stress, tiefes Atmen, Fantasiereisen, Musik, Kangarooing bei Früh- und Neugeborenen etc.)

- Positionierung (durch Bewegung, Lagerung, Wärmeoder Kälteanwendung etc.) [4][9][15]

Neben den zentralen schmerzlindernden Maßnahmen werden im Behandlungsplan auch die individuellen Ziele des Kindes und der Familie thematisiert. Fragen nach dem akzeptablen Schmerzmaß, nach Tagesrhythmus, angestrebten Freizeitaktivitäten oder notwendigen Schlafzeiten sollten diskutiert und wenn möglich gemeinsam beantwortet werden. Hierbei steht die Integration des Behandlungsplans in den Lebensalltag des Kindes und seiner Familie im Fokus und nicht umgekehrt [13].

\section{Beratung}

Ein weiterer Baustein im Rahmen des Schmerzmanagements ist das Thema Beratung, Anleitung und Schulung. Wie in jedem kindspezifischen Pflegekontext steht auch hier die Familienorientierung im Mittelpunkt. Eltern sind die Experten für ihr Kind, und vor allem im häuslichen Alltag kann die professionelle Pflege eines Kindes oder Ju- 
gendlichen nur gemeinsam mit ihnen gestaltet werden. So müssen Eltern befähigt werden, Assessments zur Schmerzeinschätzung anzuwenden, den Schmerzverlauf zu kontrollieren und zu beurteilen, Nebenwirkungen der Medikamente zu erkennen oder nicht-medikamentöse Maßnahmen selbst durchzuführen. Von großer Wichtigkeit ist die Aufklärung über die in der breiten Gesellschaft immer noch bestehenden Mythen der Opioidtherapie, um Ängste und Ressentiments abzubauen und den Kindern dadurch eine adäquate schmerzlindernde Option zu ermöglichen [13].

Welche zentrale Rolle ein aktueller evidenzbasierter Wissensstand der Pflegenden im Rahmen eines Schmerzmanagements bei Kindern und Jugendlichen - insbesondere im Bereich der Beratung und Schulung - einnimmt, wird durch die bisherigen Ausführungen deutlich nachvollziehbar und muss nicht weiter erläutert werden.

\section{Schlussbemerkungen}

Schmerz bei chronisch kranken, pflegebedürftigen Kindern und Jugendlichen mit und ohne Behinderung ist ein häufig auftretendes Pflegeproblem und fordert auch in der Praxis der häuslichen Kinderkrankenpflege ein reflektiertes, systematisches, evidenzbasiertes und vor allem multidisziplinäres Vorgehen.

Trotz zunehmender Fortschritte steckt jedoch die tatsächliche Umsetzung in der Praxis immer noch in den Kinderschuhen - zu oft werden Schmerzen von Kindern fehlinterpretiert, ignoriert, bagatellisiert oder therapeutisch mit Sedativa anstatt mit Analgetika behandelt.

Seitens der Pflegenden ist hier Mut gefragt, die professionelle pflegerische Kompetenz innerhalb der eigenen Berufsgruppe, aber vor allem im multidisziplinären Kontext zu vertreten und im Sinne einer positiven kindlichen Entwicklung auch dafür zu kämpfen.

\section{Autorinnen/Autoren}

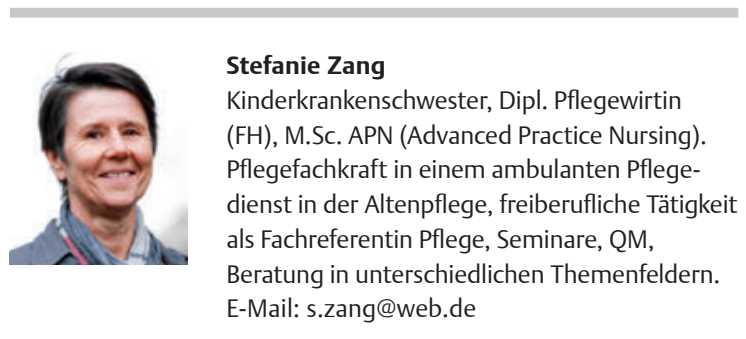

Literatur

[1] Gottschling S. Besonderheiten der Schmerztherapie bei Kindern. Zentrum für Kinderschmerztherapie und Palliativmedizin, Klinik für Kinder- und Jugendmedizin der Universität des Saarlandes; o.J.
[2] Boettcher M, Göttler S, Stoffel L et al. Schmerzmanagement bei Kindern in der Schweiz. Monatsschrift Kinderheilkunde 2012, 160(9): 1-6

[3] Anand KJS, Hickey PR. Pain and its effects in the human neonate and fetus. N Engl J Med 1987, 317(21): 1321-9

[4] Deutsches Netzwerk für Qualitätsentwicklung in der Pflege (DNQP, Hrsg.) Expertenstandard Schmerzmanagement in der Pflege bei akuten Schmerzen. 1. Aktualisierung 2011 einschließlich Kommentierung und Literaturstudie. Hochschule Osnabrück; 2011

[5] Wager J. Education Day „Schmerz“. Akuter und chronischer Schmerz: Unterschiede und Gemeinsamkeiten. Deutsches Kinderschmerzzentrum, Vestische Kinder- und Jugendklinik Datteln der Universität Witten Herdecke; 2017

[6] Deutsches Netzwerk für Qualitätsentwicklung in der Pflege (DNQP, Hrsg.) Expertenstandard Schmerzmanagement in der Pflege bei chronischen Schmerzen. Entwicklung - Konsentierung - Implementierung. Hochschule Osnabrück; 2015

[7] Deutsches Kinderschmerzzentrum. Chronische Schmerzerkrankung im Kindes- und Jugendalter, 2015. Online unter www.deutsches-kinderschmerzzentrum.de/aerzte-undtherapeuten/chronische-schmerzen-im-kindes-undjugendalter, letzter Zugriff 14.11.2018

[8] Ellert U, Neuhauser H, Roth-Isigkeit A. Schmerzen bei Kindern und Jugendlichen in Deutschland: Prävalenz und Inanspruchnahme medizinischer Leistungen. Ergebnisse des Kinder- und Jugendgesundheitssurveys (KIGGS). Bundesgesundheitsblatt - Gesundheitsforschung - Gesundheitsschutz 2007, 50(5/6): 711-7

[9] Bundesverband Häusliche Kinderkrankenpflege (BHK) e. V. Schmerzmanagement in der ambulanten Kinderkrankenpflege bei akuten Schmerzen; 2015

[10] Zernikow B, Blankenburg M, Dubbel G et al. Schmerztherapie bei Kindern mit lebenslimitierenden, nichtonkologischen Erkrankungen. Kinderärztliche Praxis 2009, 80(4): 256-70

[11] Zernikow B, Hechler T. Schmerztherapie bei Kindern und Jugendlichen. Deutsches Ärzteblatt 2017, 105(28-29): 511-22

[12] de Kuiper M. Schmerz und Schmerzmanagement bei Kindern. Wiesbaden: Ullstein Medical Verlag; 1999

[13] Bundesverband Häusliche Kinderkrankenpflege (BHK) e.V. Schmerzmanagement in der ambulanten Kinderkrankenpflege bei chronischen Schmerzen; 2016

[14] McCaffery M, Beebe A, Latham, J. Schmerz. Ein Handbuch für die Pflegepraxis. Deutsche Ausgabe herausgegeben von Jürgen Osterbrink. Wiesbaden: Ullstein Mosby; 1997

[15] Chanton A, Jerjen P. Nicht-medikamentöse Interventionen zur Schmerzbehandlung von Kindern. Eine systematische Literaturübersicht. Diplomarbeit. Hochschule Wallis für Gesundheit und soziale Arbeit; 2006

Bibliografie

DOI https://doi.org/10.1055/a-0792-9194

JuKiP 2019; 8: 20-23

(c) Georg Thieme Verlag KG Stuttgart · New York ISSN 1439-2569 